\title{
Black-Hole Numbers of Iteration of Digital Quintic Power Sum
}

\author{
Yao Zhang ${ }^{1, a}$, Xiaopan $\mathrm{Li}^{1, \mathrm{~b}}$ and Wenliang $\mathrm{WU}^{1, \mathrm{c}, \text { * }}$
}

${ }^{1}$ College of Physics and Electronic Engineering, Zhaotong University, Zhaotong 657000, China aemail: 237763661@qq.com, bemail:Ixpzrc@163.com, bemail:zhsswwl@163.com *corresponding author

Keywords: Black-hole Number, Sum of Digital Power, Iteration, Fixed Point, Periodic Point

\begin{abstract}
For iteration of quintic power sum of digits there are seven fixed points, two 2-circles, one 4-circle, one 6-circle, two 10-circles, one 12-circle, one 22-circle and one 28-circle. The probabilities for natural numbers falling into each fixed point or circle by several times iterating of quintic power sum are unbalanced. In it about $1 / 3$ fall into 22-circle, about 1/4 into 28-circle, the probability of falling into 12 -circle is slightly less than $1 / 4$, that of falling into seven fixed points is less than $1 \%$.
\end{abstract}

\section{Introduction}

The black-hole number problem is a topic of conversation that is extensively paid close attention to recently. Paper [1] gives out periodic points and fixed points of iteration of quartic power sum of digits, paper [2] also mention it. This paper studies periodic points and fixed points of iteration of quintic sum of digits based on paper [1].

\section{Lemma}

Natural number and its iteration of quintic power sum of digits are congruent with respect to module 3.

Suppose natural number $n=\sum_{i=0}^{m} a_{i} \times 10^{i}=\sum_{i=0}^{m}\left(3 b_{i}+c_{i}\right) \times 10^{i}$, where $b_{i} \in\{0,1,2,3\}, \quad c_{i} \in\{1,-1,0\}$.

Assume $f(n)=\sum_{i=0}^{m} a_{i}^{5}$, since

$$
\begin{gathered}
n \equiv \sum_{i=0}^{m} a_{i} \times 10^{i} \bmod 3 \equiv \sum_{i=0}^{m} a_{i} \times(9+1)^{i} \bmod 3 \equiv \sum_{i=0}^{m} a_{i} \bmod 3 \equiv \sum_{i=0}^{m} c_{i} \bmod 3, \\
f(n) \equiv \sum_{i=0}^{m} a_{i}^{5} \bmod 3 \equiv \sum_{i=0}^{m}\left(3 b_{i}+c_{i}\right)^{5} \bmod 3 \equiv \sum_{i=0}^{m} c_{i}^{5} \bmod 3 \equiv \sum_{i=0}^{m} c_{i} \bmod 3,
\end{gathered}
$$

therefore $f(n) \equiv n \bmod 3$. The proof is completed.

From proof of the Lemma, its conclusion can be generalized to iteration of sum of digital arbitrary odd power.

\section{Theorem}

Iteration of quintic power sum of digits have and only have following periodic points and fixed points:

Seven fixed points: 1, 4150, 4151, 54748, 92727, 93084 and 194979, two 2-circles (2 periodic points): (58618,76438), (89883,157596), one 4-circle (4 periodic points): (10933, 59536, 73318, 50062), one 6-circle (6 periodic points): (8299, 150898, 127711,33649, 68335, 44155), two 10-circles (10 periodic points):(8294, 92873, 108899, 183635, 44156,12950, 62207, 24647, 26663, 23603)and(9044, 61097, 83633, 41273, 18107, 49577, 96812, 99626, 133682, 41063), one 12-circle (12 periodic points): (24584, 37973, 93149, 119366, 74846, 59399, 180515, 39020, 59324, 63473, 26093, 67100), one 22-circle (22 periodic points): (9045, 63198, 99837, 167916, 91410, 
60075, 27708, 66414, 17601, 24585, 40074, 18855, 71787, 83190, 92061, 66858, 84213, 34068, 41811, 33795, 79467, 101463), one 28-circle (28 periodic points): (244, 2080, 32800, 33043, 1753, 20176, 24616, 16609, 74602, 25639, 70225, 19996, 184924, 93898, 183877, 99394, 178414, 51625, 14059, 63199, 126118, 40579, 80005, 35893, 95428, 95998, 213040, 1300).

\section{Proof}

First, since $9^{5}=59049$, then quintic power sum of digits of each six-bit number is less than $9^{5} \times 6=354294$. In other words, arbitrary natural number that greater than 354294 , by one iteration of quintic power sum, the conclusion is must less than itself. So we only need to verify the theorem by computing natural numbers that less than 354294.

Second, based on commutative law of addition, the conclusion of iteration of $n$-power sum of digits is uncorrealated with the order of digits. So it doesn't need to compute all natural numbers that greater than 354294, it is practicable only to compute numbers that its higher bit less than or equal to lower one within 354294.

By iterating 4416 positive integers that satisfied with conditions mentioned above, we obtain following conclusions:

1. Only trivial 1 fall into fixed point 1 . It is congruent with 3 with respect to module 1 .

2. Only 1145 fall into fixed point 4151 and by only one iterating. It is congruent with 3 with respect to module 2 .

3. Only 22779 fall into fixed point 92727 and only147999 fall into fixed point 194979 by one iterating. They are congruent with 3 with respect to module 0 .

4. Three numbers: 145, 244456 and 335677 fall into fixed point 4150, by one, two, two iterating respectively. They are congruent with 3 with respect to module 1 .

5. Three numbers: 888, 3489 and 334668 fall into fixed point 93084, by two, one, two iterating respectively. They are congruent with 3 with respect to module 0 .

6. There are 22 numbers falling into fixed point 54748: $\{44578\},\{13669,35677\},\{12358$, 114577, 122227, 123379, 223369\}, \{55579, 125566, 133666, 228889, 233578, 235777, 333478\}, $\{13678\},\{247,11227,244477,255889,256699\},\{11113\}$ 。 They are congruent with 3 with respect to module 1 and each group fall into fixed point 54748 by one to seven iterating respectively.

7. Only two numbers 38889 and 155679 fall into 2-circle $(89883,157596)$ by one iterating. They are congruent with 3 with respect to module 0 .

8. There are 161 numbers falling into 2-circle $(58618,76438)$. They can be divided into 29 groups: $\{15688,34678\}$; $\{1447,11899,24688,24778,34777,35668,112789,144559,144679$, 245578, 333577, 334678, 344488\}; \{199, 1189, 12679, 22447, 22456, 23488, 34789, 36778, 111778, 133459, 144448, 145666, 145999, 156778, 247777, 256678, 333358, 333688\}; \{2479, 4999, 13477, 23446, 35557, 111337, 112999, 124468, 167788, 334666, 334999\}; \{3688, 13459, 139999, 166678, 245689, 346888\}; \{799, 23335, 45799, 227779, 244678, 334489\}; \{12589, 111178, 255688\}; \{23566, 25789, 111289, 112366, 112699, 123589, 125779, 133567, 233347\}; \{4477, 22288, 22699, 34567, 55666, 112489, 114559, 133699, 134449, 222499, 233389\}; \{23599, 112669, 222568, 224899, 335566\}; \{139\}; \{1156, 1225\}; \{115555, 123367, 145678, 222457, 258889, 344668\}; \{26899\}; \{2569, 34588\}; \{457, 3367, 11467, 122668, 123349, 222445\}; $\{12238$, 22237, 67777, 78889, 124558, 126889, 234688\}; \{889, 4699, 27778, 46666, 49999\}; \{11278, 115669, 223345\}; \{5689, 24499, 44446, 134569, 145588\}; \{589, 2446, 13588, 15778, 24469, 116668, 144799, 222355, 223336\}; \{2359, 112267, 112345, 122245, 133777, 134458, 145567, 145669, 234466, 246679\}; \{7789, 11557, 112558, 113389, 123448, 134899, 244447, 257779, 333889, 344599\}; \{5599, 335599\}; $\{478\} ;\{133,226\} ;\{111115,133339\} ;\{7888,113899\}$; $\{123499\}$. Each group fall into the 2-circle by one to twenty-nine iterating respectively and they are congruent with 3 with respect to module 1.

9. There are 102 numbers falling into 4-circle (10933, 59536, 73318, 50062). They can be divided into 14 groups: \{256, 1339, 13378, 35569\}; \{55, 1147, 2239, 11188, 12445, 111478, 124477, 222559\}; \{2233, 11344, 14677, 22357, 26689, 26779, 44455, 46888, 47788, 115558, 
125899, 145888, 155599, 224557, 235888, 236779, 244789, 245599, 255799, 333334, 347788\}; \{2344, 2578, 13666, 24679, 33577, 37777, 45688, 66688, 111169, 112225, 113557, 225559, $337777\}$; $\{5788,11599,22267,33778,114679,122278,135559,223777,225568,335779$, 344899\}; $\{17788,112456,133669,233566\} ;\{24889,45679,115588\} ;\{178,13399,15568,22378$, 122479, 166777, 222469, 266677\}; \{157, 1126, 4567, 11266, 134779, 135679, 235669, 235699, 245557\}; \{37, 124, 268, 122449, 122569, 133333, 223366, 233356\}; $\{4,223,1333,33349,36799$, $111112\}$; $\{1111,1138,112234\} ;\{55699,233689,235567\} ;\{112339\}$. Each group fall into the 4-circle by one to fourteen iterating respectively and they are congruent with 3 with respect to module 1.

10. There are 74 numbers falling into 6-circle (8299, 150898, 127711, 33649, 68335, 44155). They can be divided into 10 groups: $\{2899,14455,15889,33469,33568,111277\}$; $\{1477,2248$, 4777, 5668, 5677, 12457, 16777, 22999, 55888, 111679, 113779, 122677, 222577, 234478, 234667\}: $\{14557,25558,35578,77779,113488,223357,244588,246778,333388,345688$, 345778\}; \{4678, 5578, 22678, 25777, 66799, 112678, 145777, 155668, 167899, 245677, 245899, 333556\}; $\{7777,114778,115678,123478,233569,235666,277777,334459\}:\{16,1999,45889$, 135799, 136999, 334699, 336889\}; \{13447, 13888, 13999, 22222, 45589, 111124, 134668, 155788\}; \{23668, 166666, 268888, 335788\}: \{778, 112228\}; \{11116\}. Each group fall into the 6 -circle by one to ten iterating respectively and they are congruent with 3 with respect to module 1 .

11. There are 263 numbers falling into 10-circle (8294, 92873, 108899, 183635, 44156, 12950, 62207, 24647, 26663, 23603). They can be divided into 16 groups: $\{1259,2267,2336,2489,14456$, 18899, 23666, 23789, 24467, 133568\}; \{446, 1466, 1667, 2459, 2666, 11378, 13667, 22259, 22367, 23567, 26669, 27779, 27899, 45557, 55556, 56888, 116669, 122228, 123479, 123569, 124568, 126788, 134579, 144566, 223667, 244457, 334478, 336779\}; \{47, 899, 1889, 2789, 3347, 3356, 3359, 6899, 14777, 15668, 15677, 22268, 22379, 25556, 34445, 34478, 35666, 44666, 55778, 111779, 114578, 124799, 133577, 144446, 146777, 156668, 156677, 166688, 222239, 223469, 224477, 233459, 244466, 255569, 333356\}; \{179, 11348, 13346, 14447, 16688, 22466, 22778, 23444, 33338, 33458, 44558, 45566, 111446, 111488, 112478, 112568, 115589, 117899, 122477, 124559, 134459, 134999, 144479, 155789, 233378, 233567, 233999, 255899, 267899, 333389, $344444\}$; $\{77,3677,122468,124667,135557,177788,225557,334457\} ;\{2888,4667,4778,5678$, 11789, 12668, 13355, 13577, 14459, 14888, 15569, 16799, 22346, 23345, 23999, 25889, 33488, 33689, 113468, 114788, 118889, 123779, 147899, 166889, 188888, 223379, 224489, 224888, 225566, 227789, 255578, 333449, 334667, 344699\}; \{89, 338, 368, 1388, 1469, 13337, 14666, 22337, 26678, 33557, 47999, 56999, 111179, 111257, 112226, 113477, 113999, 125588, 233588, 234566, 256778, 337889\}; \{1169, 1688, 7799, 11222, 11678, 12227, 22349, 24455, 24689, 34577, 111278, 111599, 111689, 112778, 122588, 123677, 133349, 233579, 257999, 333347, 333566\}; $\{17,227,14999,22499,45788,46679,56666,111566,114689,125789,145556,234677,244499$, 256667, 267779, 334466\}; \{2357, 25688, 34559, 34778, 44669, 55568, 67889, 115568, 126677, 222488, 235889, 268889\}; \{25679, 557, 3788, 22445, 45677, 111578, 145778, 222467, 244445, 255788, 277799\}; \{23579, 44567, 55688, 123335, 123455, 123578, 167777, 222578, 244478, 346679\}; \{11258, 111239, 113567, 135899, 145889, 224999, 244688, 266678\}; \{1499, 38999, 66779, 67799, 146999, 222599, 226688, 245789\}; \{16679, 35669, 134489, 222479, 226667\}; \{12488, 222227, 222269, 346799\}. Each group fall into the 10-circle by one to sixteen iterating respectively and they are congruent with 3 with respect to module 2 .

12. There are 239 numbers falling into 10-circle(9044, 61097, 83633, 41273, 18107, 49577, 96812, 99626, 133682, 41063). . They can be divided into 19 groups: \{449, 1178, 1346, 1679, 12347, 12689, 26699, 33368, 45779, 123368\}; \{2255, 3449, 4445, 11126, 12566, 12779, 16667, 34688, 35555, 35678, 45689, 111335, 112256, 122234, 122555, 123788, 134555, 222347, 223334, 224555, 335555\}; \{68, 11345, 12599, 14678, 15788, 22247, 24488, 26888, 38888, 45578, 123668, 146678, 155555, 337778, 344459, 345788, 346889, 347777\}; \{1247, 8999, 11447, 14579, 33677, 58889, 111122, 111227, 117788, 133556, 223355, 226778, 335999\}; \{1556, 4577, 13556, 16778, 22688, 25589, 55559, 112229, 112577, 126689, 126779, 233333, 237779, 334568\}; \{566, 1268, 5588, 25667, 156779, 225779, 233477, 236888, 245555, 334556, 347789\}; \{134, 2288, 5579, 
13349, 13466, 14669, 23678, 36779, 116888, 133445, 144467, 155777, 266777, 299999\}; \{8, 1235, 2444, 3368, 3377, 6677, 12788, 13568, 23477, 24479, 44468, 66668, 111677, 112223, 122348, 124577, 144449, 244559, 333359, 333557, 345779\}; \{5, 35, 599, 2345, 13358, 22577, 33449, 33578, 34448, 44888, 77789, 111377, 112238, 112277, 112388, 157778, 269999, 345557\}; \{377, 1112, 2678, 3344, 11111, 11777, 12248, 55889, 115889, 122225, 122999, 124448, 144677, 179999, 235799, 244667, 245999, 248888, 335777\}; \{1238, 12224, 12455, 112235, 222335, 222455, 225578, 233399, 234599, 236999, 247889, 266789\}; \{128, 1115, 15566, 155699, 223799, 335789\}; \{1136, 1244, 1334, 2222, 11114, 124478, 277778\}; \{335, 11138, 12245, 12449, 22244, 23348, 33356, 78899, 111125, 116666, , 346667\}; \{248, 299, 788, 11228, 17888, 37778, 111668, , $112559\}$; $\{26,44,2447,12368,23399,46688,56789,57779,111113,112457,144899,222446$, 233336\}; \{15557, 23558, 25799, 115679, 125669, 134669, 136778, 146789, 222257, 224669, 233678, 234458, 234488, 245579, 255557, 333779\}; \{134888, 155666, 222779, 225788, 237899, $346688\}$; $\{68888\}$ 。 Each group fall into the 10-circle by one to nineteen iterating respectively and they are congruent with 3 with respect to module 2 . A interesting fact is that most of them at first become 83633 in the 10 -circle.

13. From Lemma, in remaining numbers there are 967 numbers falling into 12-circle (24584, 37973, 93149, 119366, 74846, 59399, 180515, 39020, 59324, 63473, 26093, 67100). There are congruent with 3 with respect to module 2. The greatest number of times of iteration is for 111266, by 57 times iterating and then become 24584 in the 12-circle. There are 1107 numbers falling into 28-circle(244, 2080, 32800, 33043, 1753, 20176, 24616, 16609, 74602, 25639, 70225, 19996, 184924, 93898, 183877, 99394, 178414, 51625, 14059, 63199, 126118, 40579, 80005, 35893, 95428, 95998, 213040, 1300). There are congruent with 3 with respect to module 1 . The greatest number of times of iteration is for 134566, by 44 times iterating and then become 70225 in the 28-circle. There are 1469 multiples of 3 and they fall into 22-circle (9045, 63198, 99837, 167916, 91410, 60075, 27708, 66414, 17601, 24585, 40074, 18855, 71787, 83190, 92061, 66858, 84213, $34068,41811,33795,79467,101463)$. The greatest number of times of iteration is for 16851, by 56 times iterating and then become 9045 in the 22-circle. The proof is completed.

\section{Summary}

From proof of the theorem, as that expounded in paper [1], the probabilities of falling into each fixed point or periodic point by several times iterating of quintic power sum of digits are unbalanced. In it abot1/3 fall into 22-circle, about $1 / 4$ into 28-circle, the probability of falling into 12 -circle is slightly less than $1 / 4$, that of falling into severn fixed points is less than $1 \%$.

\section{Acknowledgement}

This work is supported by the Youth Program of the the Applied Basic Research Programs of Science and Technology Department of Yunnan Province, China (Project No. 2013FD056).

\section{References}

[1] Song Zhaoshou, Zhang Yao, Wu Wenliang. The Periodic Points of Iteration of Each Digit's Biquadratic Sum of Natural Number [J]. Journal of Chongqing University of Science and Technology(Natural Sciences Edition), 2012 (4) 177-178.

[2] G. Richard, Unsolved Problems in Number Theory, 2nd ed., Springer Verlag, New York, 1994.

[3] Shi Kefu, Wang Mingqiang. On The Sum of Each Digit'S Square of Integer [J]. Journal of Qufu Normal University(Natural Science), 1999 25(4) 45-46.

[4] Liu Rong-hua, Zhu Wei. Several Conclusions about Black-Hole Numbers [J] . Journal of Changchun Teachers College, 2005 24(1) 24-26. 